\title{
Wisdom of crowds: much ado about nothing
}

\author{
Sandro M. Reia ${ }^{1}$ and José F. Fontanari ${ }^{1}$ \\ ${ }^{1}$ Instituto de Física de São Carlos, Universidade de São Paulo, \\ Caixa Postal 369, 13560-970 São Carlos, São Paulo, Brazil
}

\begin{abstract}
The puzzling idea that the combination of independent estimates of the magnitude of a quantity results in a very accurate prediction, which is superior to any or, at least, to most of the individual estimates is known as the wisdom of crowds. Here we use the Federal Reserve Bank of Philadelphia's Survey of Professional Forecasters database to confront the statistical and psychophysical explanations of this phenomenon. Overall we find that the data do not support any of the proposed explanations of the wisdom of crowds. In particular, we find a positive correlation between the variance (or diversity) of the estimates and the crowd error in disagreement with some interpretations of the diversity prediction theorem. In addition, contra the predictions of the psychophysical augmented quincunx model, we find that the skew of the estimates offers no information about the crowd error. More importantly, we find that the crowd beats all individuals in less than $2 \%$ of the forecasts and beats most individuals in less than $70 \%$ of the forecasts, which means that there is a sporting chance that an individual selected at random will perform better than the crowd. These results contrast starkly with the performance of non-natural crowds composed of unbiased forecasters which beat most individuals in practically all forecasts. The moderate statistical advantage of a real-world crowd over its members does not justify the ado about its wisdom, which is most likely a product of the selective attention fallacy.
\end{abstract}

\section{INTRODUCTION}

The wisdom of crowds usually refers to the notion that a collection of individuals - the crowd - can solve problems better than most individuals within it, including experts [1]. The idea was brought to light by Galton's 1907 analysis of a contest to guess the weight of an ox at the West of England Fat Stock and Poultry Exhibition in Plymouth 2, 3. Despite being more than a century old, the wisdom of crowds is still a subject of fascination for laypeople and scientists as well. This fascination stems from reports of the remarkably accurate appraisal produced by the statistical average of independent estimates of the magnitude of an unknown quantity. For instance, in the ox-weighing contest, the crowd overestimated the weight of the ox by less than $1 \%$ of the true weight 2 .

There are a few attempts to explain the wisdom of crowds using purely statistical arguments. The first idea that comes to mind is that the individual estimates are unbiased, that is, that the errors spread in equal proportion around the true value of the unknown quantity so that they cancel out when the estimates are combined together 4, 5]. Although it is hard to accept the nonexistence of systematic errors on the individual estimates, this explanation is rather popular perhaps because its underlying assumption is difficult to verify [6].

A somewhat more sophisticated explanation for the wisdom of crowds is offered by the diversity prediction theorem [7, which asserts that the error of the collective estimate is never greater than the average individual error. Moreover, as hinted by its name, the theorem has a say in the role of the diversity of the individual estimates. In fact, since the theorem asserts that the quadratic collective error equals the average quadratic individual error minus the diversity of the estimates, one is tempted to think that the increase of the diversity would improve the crowd accuracy [7.

In addition to the statistical explanations, there is a psychophysical model of the wisdom of crowds, viz., the augmented quincunx model of probabilistic cue categorization [8, which assumes that there exists a typical value of the estimated quantity that is common knowledge of the population. The real object, say Galton's ox, is then compared with the prototypical object through a number of perceptible cues that may be categorized incorrectly by the individuals. The main prediction of the model is that information about the collective error can be inferred from the skewness of the distribution of the estimates 8 .

In order to test the predictions, as well as the relevance, of the aforementioned explanations for the wisdom of crowds, we use forecasts of economic indicators that are publicly available in the Federal Reserve Bank of Philadelphia's (FRBP) Survey of Professional Forecasters 9. As this database offers quarterly projections of the economic indicators, we can tune the difficulty of the forecasts by controlling for the forecast range. We find that the collective error and the diversity of the estimates are affected significantly by that range. Most interestingly, we find that the difficulty of the forecast is typically associated with a large variance and a long left tail of the distribution of estimates.

Overall we find that the data do not support any of the proposed explanations for the wisdom of crowds. First, in opposition to the unfounded interpretation of the diversity prediction theorem, we find a positive correlation between the collective error and the diversity of estimates. Second, we find that, once the range of the forecasts is accounted for, the skewness of the distribution of estimates does not influence significantly the crowd accuracy, in opposition to the predictions of the augmented quincunx 
model. Third, we find that the unbiased estimates assumption confers on the crowd an enormous advantage over the individuals within it, which is at great variance with the data.

Our main finding, which was obtained using almost $10^{4}$ forecast experiments for several economic indicators in the FRBP forecast database, is that only rarely the crowd beats all individuals within it. More precisely, this happens in less than $2 \%$ of the forecast experiments that we analyzed. In addition, the crowd beats most individuals within it in less than $70 \%$ of our experiments, which means that there is a fair chance that an individual selected at random will perform better than the crowd. Clearly, the purely statistical advantage of the crowd over its members does not justify the high esteem it enjoys, which is most likely a product of the selective attention fallacy.

The rest of this paper is organized as follows. In section II we offer an outline of the main ideas used to explain the wisdom of crowds, viz., the diversity prediction theorem [7, the augmented quincunx model 8 and the unbiased estimates assumption [4. In section III we describe briefly the FRBP forecast database 9] from where we have extracted the forecast experiments. In section IV] we present and analyze the results of those experiments in the light of the known explanations for the wisdom of crowds. In $\mathrm{V}$ we replicate those forecast experiments using virtual unbiased agents so as to verify the predictions of the unbiased estimates assumption. In V] we revisit a few wisdom-of-crowds experiments where the participants are laypeople and show that their results are consistent with the results obtained using the expert forecasters of the FRBP database. Finally, section $\mathrm{V}$ is reserved to our concluding remarks.

\section{THREE FRAMEWORKS FOR UNDERSTANDING THE WISDOM OF CROWDS}

Here we describe briefly three frameworks that claim to explain the wisdom of crowds, viz., the diversity prediction theorem, the augmented quincunx model and the unbiased estimates assumption. These proposals make a variety of specific predictions that will be tested using the FRBP forecast database in section III

\section{A. The diversity prediction theorem}

The diversity prediction theorem is viewed as a main achievement to those allured by the idea that the performance of groups can be boosted by increasing the diversity of their members [7, although even simple agentbased models indicate that the effects of diversity can be rather unpredictable in nontrivial problem-solving scenarios [10]. This theorem shows that the quadratic collective error is related in a very simple manner to the average quadratic individual error and to a measure of the diversity of the estimates. More pointedly, let us denote by $g_{i}$ the estimate of some unknown quantity by individual $i=1, \ldots, N$, and by $G$ the true value of the unknown quantity. The collective estimate is defined as the arithmetic mean of the individual estimates, that is,

$$
\langle g\rangle=\frac{1}{N} \sum_{i=1}^{N} g_{i},
$$

as usual. We note, however, that Galton used the median of the individual estimates as the crowd estimate in his seminal ox-weighing experiment [2, though the arithmetic mean proved to be a much better estimator in that case 3 . Thus, the collective error is defined as the signed quantity

$$
\gamma=G-\langle g\rangle
$$

In most of this paper, however, we consider the unsigned collective error $|\gamma|$; the only exception is the discussion of the prediction of the augmented quincunx model that the signs of $\gamma$ and of the skewness of the distribution of estimates are negatively correlated. Defining the average quadratic individual error as

$$
\epsilon=\frac{1}{N} \sum_{i=1}^{N}\left(g_{i}-G\right)^{2}
$$

and the diversity or variance of the estimates as

$$
\delta=\frac{1}{N} \sum_{i=1}^{N}\left(g_{i}-\langle g\rangle\right)^{2},
$$

we have the identity

$$
\gamma^{2}=\epsilon-\delta
$$

which is Page's diversity prediction theorem [7]. It asserts that the quadratic collective error equals the average quadratic individual error minus the prediction diversity. This result is sometimes viewed as indication that the increase of the prediction diversity $\delta$ results in the decrease of the quadratic collective error $\gamma^{2}$. Of course, since $\delta$ and $\epsilon$ cannot be varied independently of each other, this interpretation is not correct. Nevertheless, the relationship, if any, between the diversity of the estimates and the collective error is a very interesting issue that can be investigated using a large number of equivalent forecast experiments, which is the approach we follow in this paper. We note that the diversity of estimates $\delta$ is known in the statistical literature as the precision of the estimates, that is, the closeness of repeated estimates (of the same quantity) to one another [11.

We stress that the diversity prediction theorem guarantees only that the quadratic collective error $\gamma^{2}$ is never greater than the mean quadratic individual error $\epsilon$. As we will see in section III, this is not a very useful result from the practical perspective since, for instance, it does 
not imply that the crowd is better than most individuals. In particular, we will report an experiment where the collective estimate is worse than the estimate of about $85 \%$ of the individuals. Hence, $\gamma^{2} \leq \epsilon$ does not imply that it is always advantageous to favor the collective estimate over the estimate of a randomly chosen individual in the group.

As an amusing side note, we mention the resemblance between the discussions about the value of the diversity prediction theorem and the arguments about the relevance of the celebrated Price equation for evolutionary biology [12. We note that Price's equation, which has a straightforward derivation from the definition of fitness, is considered by many researchers as a mere mathematical tautology that has no predictive value at all [13].

It is not possible to investigate the influence of the diversity of the estimates on the collective error using a single forecast experiment since the values of $\gamma, \epsilon$ and $\delta$ are fixed for a particular experiment. The solution is to consider a large ensemble of roughly equivalent experiments and to look at the correlations between those quantities. This can be achieved artificially by selecting random subsamples of the estimates of a single experiment to produce many virtual experiments with fewer estimates than the original one [14]. The problem with that approach is that the resulting virtual experiments are not independent. Here we use the FRBP forecast database to collect the independent forecast experiments necessary for the correlation analysis. We note that since the collective estimate $\langle g\rangle$ and the true value $G$ may have different values for different experiments, it is necessary to introduce the dimensionless quantities $\gamma / G, \epsilon^{1 / 2} / G$ and $\delta^{1 / 2} /\langle g\rangle$ to properly compare the experiments.

\section{B. The augmented quincunx model of judgment}

The augmented quincunx is a psychophysical model of probabilistic cue categorization [8], whose name was inspired by a probability device invented by Galton in 1873 to demonstrate the central limit theorem [15. The basic idea behind this model is the assumption that there is a typical value of the unknown quantity that is common knowledge gained through experience. For instance, in the ox-weighing experiment, it is assumed that the population (or at least the participants of the contest) share the knowledge that the typical weight of oxen is $\hat{G}$. In order to estimate the weight $G$ of a particular ox, the contestants focus on a number of perceptible cues $c=1, \ldots, C$ that are correlated with $G$ (e.g., the height of the ox or the degree to which its ribs are showing). If a cue $c$ indicates that the ox is heavier (lighter) than the prototype ox then the typical weight is increased (decreased) by a factor $\eta_{c}$. Hence

$$
G=\hat{G}+\sum_{c=1}^{C} \eta_{c}
$$

where $\eta_{c}$ can be positive or negative depending on the correlation between cue $c$ and the ox weight. Stochasticity enters the augmented quincunx model because the contestants can perceive a cue incorrectly. More pointedly, the estimate of contestant $i$ is

$$
g_{i}=\hat{G}+\sum_{c=1}^{C} u_{c} \eta_{c}
$$

where $u_{c}$ is a random variable that takes on the value +1 with probability $p$ and the value -1 with probability $1-p$ 8. This means that a cue is perceived correctly with probability $p$ and incorrectly with probability $1-p$. In particular, if individual $i$ can perceive all cues correctly (i.e., $p=1$ ) then its estimate is perfect (i.e., $g_{i}=G$ ), despite the fact that $\hat{G} \neq G$. For simplicity, the model assumes that all contestants are equivalent, i.e., the cue categorization probability $p$ is the same for all individuals.

Although $\hat{G}, C$ and $\eta_{c}$ are hard to access quantities, the augmented quincunx model makes some interesting general predictions. For instance, the model predicts that the collective error $\gamma$ can be inferred from the degree and direction of the skewness of the distribution of individual estimates: estimate distributions have greater negative (positive) skew when the mean estimate $\langle g\rangle$ underestimates (overestimates) the true value $G$ by greater margin [8]. This claim means that there is a negative correlation between $\gamma / G$ and the skewness $\mu_{3}$. We recall that the skewness $\mu_{3}$ of a distribution is a dimensionless measure of its asymmetry, which is defined as

$$
\mu_{3}=\frac{1}{N} \sum_{i=1}^{N}\left(\frac{g_{i}-\langle g\rangle}{\delta^{1 / 2}}\right)^{3},
$$

where $\delta$ is the sample variance of the estimates defined in equation (4) and $\langle g\rangle$ is the mean of the estimates defined in equation (1). A negative value of $\mu_{3}$ implies that the left tail of the distribution of estimates is longer than the right tail, whereas a positive $\mu_{3}$ indicates a right-tailed distribution.

\section{The unbiased estimates assumption}

A natural explanation for the wisdom of crowds involves the well-known fact that the combination of unbiased and independent estimates guarantees the accuracy of the statistical average, provided the number of estimates is large (see, e.g., 4, 5]). In other words, if the estimates made by numerous different people scatter symmetrically around the truth, then the collective estimate is likely to be very accurate. Of course, the trouble with this explanation is the assumption that the individual estimates are unbiased, that is, that their means coincide with the true value of the unknown quantity. If correct, this assumption would imply that one could harvest the benefits of the wisdom of crowds by asking 
a single individual to make several estimates at different times (see, e.g., [16]). The unbiased estimates assumption is the limit of the augmented quincunx model where $\hat{G}=G$ and $p=1 / 2$.

In V we use the unbiased estimates assumption as a null hypothesis and replicate our analysis of the FRBP forecast experiments by replacing the expert economists by virtual unbiased forecasters. We stress, however, that ordinary people are not unbiased forecasters. On the contrary, unbiased forecasters are the ultimate experts because their estimates fluctuate symmetrically (on the average) around the true value of the quantity being estimated, i.e., they somehow 'know' its true value. In that sense, an unbiased forecaster is a theoretical construct. As a side comment, we note that the judgment of ordinary people is biased in several ways. For instance, there is the so-called anchor effect, in which mentioning in passing a figure, say 400, to a group of randomly picked individuals who are asked to guess the number of candies in a jar and a different figure, say 800, to another group exposed to the same jar leads to very distinct collective predictions [6]. Accordingly, in $\mathrm{V}$ ] we analyze the candies-in-a-jar experiment 14 and show that the resulting collective estimate obtained from the aggregation of laypeople estimates cannot be explained by the unbiased estimates assumption.

\section{THE FRBP FORECAST DATABASE}

The Federal Reserve Bank of Philadelphia's (FRBP) Survey of Professional Forecasters offers quarterly projections for five quarters of a variety of economic indicators 9. In order to lighten the analysis, here we will focus mainly on semestrial forecasts of the nominal gross domestic product (NGDP), as our conclusions apply to the other indicators as well. In particular, we consider the forecast for the current quarter, that is, the quarter when the survey was conducted and the forecasts for two and four quarters later. Henceforth we will refer to these forecasts as short-range, medium-range and long-range forecasts. We use the NGDP forecasts available in the FRBP database from the fourth quarter of 1968 to the fourth quarter of 2019 . There are $1+(2019-1969+1) \times 4=205$ quarters in total which equals the number of short-range estimate distributions. In addition, since the mediumrange forecasts predict the economic indicators two quarters after the quarter when the survey was conducted, the last two quarters of 2019 are not used for those forecasts and so the number of medium-range estimate distributions is 203. Following this reasoning, the number of long-range estimate distributions should be 201. However, the data for the long-range forecasts are missing in the first, second and third quarters of 1969, in the first quarter of 1970 and in the third quarter of 1974 , so that the number of long-range estimate distributions available in the FRBP database is 196. All forecasters are select economists and the mean number of forecasters in each
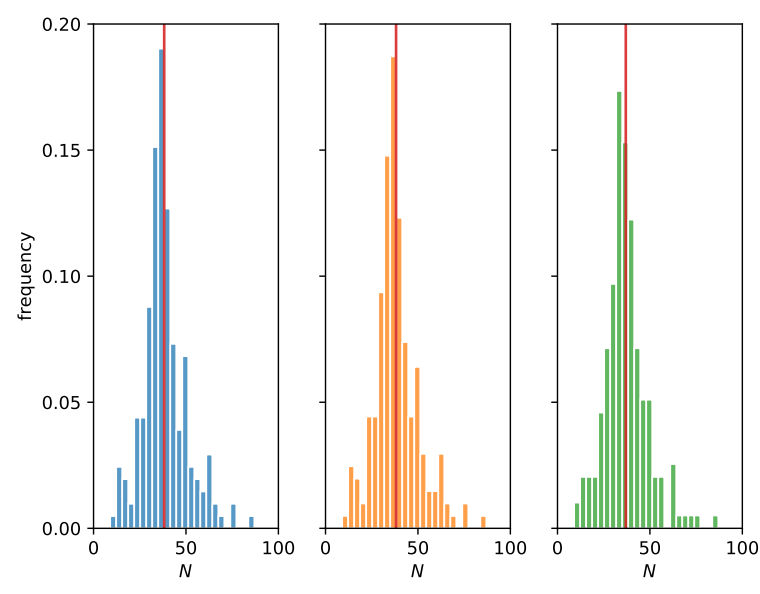

FIG. 1. Histograms of the number of participants $N$ in the 205 short-range forecasts (left panel), in the 203 mediumrange forecasts (middle panel) and in the 196 long-range forecasts (right panel) on the nominal gross domestic product of the Federal Reserve Bank of Philadelphia's Survey of Professional Forecasters. The vertical red lines indicate the average number of participants in each forecast range.

experiment is about 37 . Figure 1 shows the histograms of the number of participants $N$ in each experiment for the three forecast ranges. The minimum number of participants is $N=9$ and the maximum is $N=87$ in any forecast range.

The separation of the experiments on short-range, medium-range and long-range forecasts allows the control of the difficulty of the forecasts and the study of its influence on the distribution of the individual estimates. We note that in Ref. 8 these distinct forecast ranges were clumped together to form a single large forecast database on the NGDP. We think, however, that this procedure is not appropriate as the range of the forecasts has a major effect on the distribution of the estimates, as we will see next.

\section{RESULTS}

We begin our analysis with the study of the correlation between the scaled crowd accuracy $|\gamma| / G$ and the scaled diversity of the estimates $\delta^{1 / 2} /\langle g\rangle$. Figure 2 shows the scatter plots of these quantities for the three different forecast ranges, where each data point represents a particular forecast experiment. As already pointed out, since the number of participants $N$ as well as the NGDP true value $G$ may vary in different experiments for the same forecast range (see, e.g., figure 1), it is necessary to consider dimensionless summary statistics to properly compare the outcomes of the experiments. We recall that each experiment produces a distribution of estimates from where we extract the relevant summary statistics. For instance, figure 3 illustrates two distributions of es- 

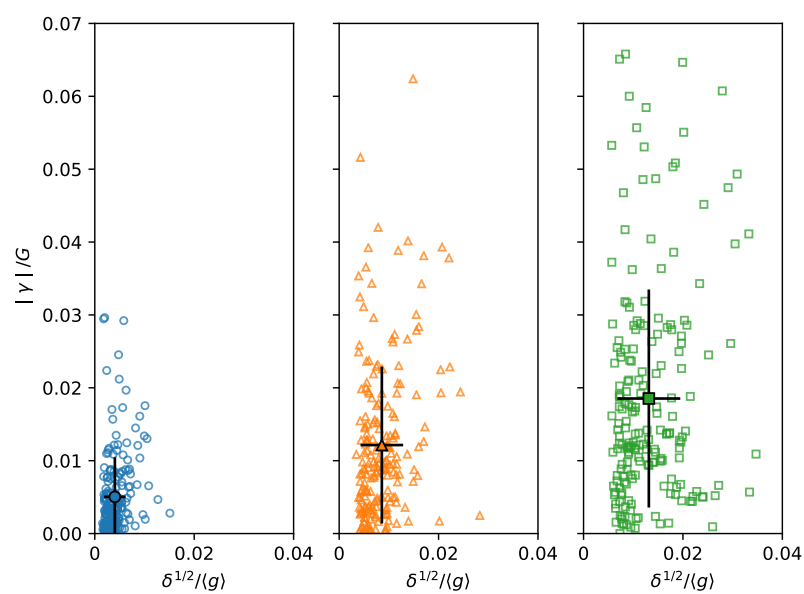

FIG. 2. Scatter plots of the scaled diversity $\delta^{1 / 2} /\langle g\rangle$ and the scaled collective error $|\gamma| / G$ for the short-range (left panel), medium-range (middle panel) and long-range (right panel) NGDP forecasts. The filled symbols and the horizontal and vertical lines indicate the means and the standard deviations.

timates for the short-range forecast scenario, which correspond to two different data points in the left panel of figure 2. The individual estimates in the $\mathrm{x}$-axis of figure 3 are scaled by the average estimate $\langle g\rangle$ defined in equation (1), which is by definition the crowd prediction, so that the scaled crowd prediction is $\langle g\rangle /\langle g\rangle=1$. Since the scaled true value is $G /\langle g\rangle$, the participants that predict better than the crowd are those whose estimates satisfy $\left|g_{i} /\langle g\rangle-G /\langle g\rangle\right|<|1-G /\langle g\rangle|$, i.e, are those whose individual errors are smaller than the error of the crowd.

Figure 2 shows that the accuracy $|\gamma| / G$ and the dispersion $\delta^{1 / 2} /\langle g\rangle$ of the estimates vary considerably as the forecast range increases, indicating that clumping those forecasts together as done in Ref. 8 may not be a judicious choice. In particular, the short-range forecasts are on the average about three times more accurate and four times less disperse than the long-range forecasts. This is somewhat expected since the farther the forecast range, the greater the odds that the predicted indicator will be influenced by unforeseen factors.

Since the two summary statistics displayed in the scatter plots of figure 2 (as well as in all scatter plots exhibited in this paper) are probably not linearly dependent, we use the nonparametric Spearman correlation coefficient $\rho$ to measure correlations rather than the usual Pearson's coefficient, which is designed to quantify linear correlations. We find, however, that these two correlation coefficients yield very similar values for the quantities considered here. In addition, the statistical significance of the measured correlation coefficients is determined by their $p$-values that yield the probability that the same coefficients are obtained if the null hypothesis is true, i.e., if the two summary statistics are uncorrelated. Hence, a very small $p$-value means that the observed correlation is very unlikely under the null hypothesis and so that it is
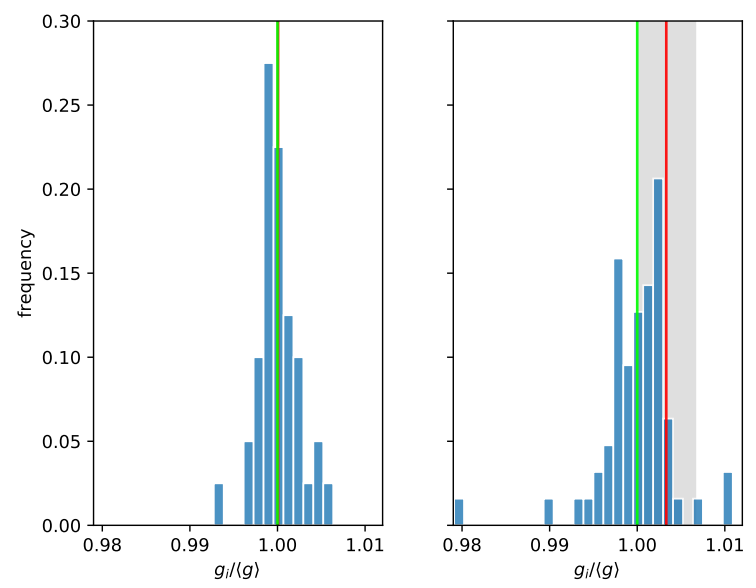

FIG. 3. Histograms of the relative estimates $g_{i} /\langle g\rangle$ for two short-range forecasts. The vertical red lines indicate the ratio between the true value of the NGDP indicator and the crowd estimate, i.e., $G /\langle g\rangle$, whereas the vertical green lines at $g_{i} /\langle g\rangle=1$ indicate the scaled crowd estimate. In the left panel there are $N=40$ participants and none of them predicted better than the crowd, whereas in the right panel there are $N=63$ participants and $43 \%$ of them predicted more accurately than the crowd. The gray region highlights the individual estimates that are better than the crowd's.

statistically different from zero.

The Spearman correlation coefficient between $\delta^{1 / 2} /\langle g\rangle$ and $|\gamma| / G$ is $\rho=0.31$ with $p$-value $<10^{-6}$ for the shortrange, $\rho=0.25$ with $p$-value $<10^{-6}$ for the mediumrange and $\rho=0.14$ with $p$-value $=0.05$ for the long-range forecasts. The unfounded interpretation of the diversity prediction theorem that associates a high prediction diversity to a low collective error implies a negative correlation between $\delta^{1 / 2} /\langle g\rangle$ and $|\gamma| / G$, which is clearly not supported by our findings. In fact, given that the forecasters are all expert economists, it is somewhat intuitive to expect that the less disperse their estimates, the closer they are to the true value. For the long-range forecast, their expertise becomes less influential to the success of the predictions and so the (statistically significant) positive correlation between collective error and diversity decreases [17]. We note that this positive correlation is a prediction of the augmented quincunx model [8] and that it holds also for the unbiased estimates assumption, as shown in $\mathrm{V}$. The reason a positive correlation between the diversity (or variance) of the estimates and the collective error is consistent with the diversity prediction theorem is because the average quadratic individual error increases together with the variance.

An interesting outcome of the augmented quincunx model refers to the role of the skewness $\mu_{3}$ of the distribution of estimates. In particular, that model predicts a negative correlation between $\mu_{3}$ and the (signed) collective error $\gamma$. Accordingly, in figure 4 we show scatter plots of $\mu_{3}$ and $\gamma / G$ as well as of $\mu_{3}$ and $\delta^{1 / 2} /\langle g\rangle$ for the different forecast ranges. The Spearman correlation 
coefficient between $\mu_{3}$ and $\gamma / G$ is $\rho=0.008$ with $p$-value $=0.91$ for the short-range, $\rho=-0.03$ with $p$-value $=0.62$ for the medium-range and $\rho=-0.12$ with $p$-value $=0.09$ for the long-range forecasts. The low values of these coefficients and their high $p$-values point to the little relevance of the skewness of the estimate distributions to the crowd prediction, in disagreement with the claims of Ref. 8. In addition, our results indicate that the skewness is weakly affected by the forecast range. This finding contrasts with the results for the dispersion of the estimates that is strongly affected by that range. In fact, our results suggest that the more difficult the forecasts, in the sense that there is more room for noise to alter the future outcome, the greater the variance of the estimates. The Spearman correlation coefficient between $\mu_{3}$ and $\delta^{1 / 2} /\langle g\rangle$ is $\rho=-0.09$ with $p$-value $=0.19$ for the short-range, $\rho=-0.36$ with $p$-value $<10^{-6}$ for the medium-range and $\rho=-0.46$ with $p$-value $<10^{-6}$ for the long-range forecasts, which implies that, at least for the medium and long-range forecasts, a large variance is associated with left-tailed estimate distributions. This interesting and statistically significant correlation is not predicted by the augmented quincunx model since the sign of the skewness is not affected by the variance in that model [8]. In addition, we note that the unbiased estimates assumption predicts no significant correlation between $\mu_{3}$ and $\delta^{1 / 2} /\langle g\rangle$ (see $\mathrm{V}$, so the negative correlation between these quantities reported here is a genuine effect of the aggregation of real-world biased forecasts.

We conclude our analysis by challenging the common view that the crowd is superior to most of its integrants 1. In fact, if people believed that an individual selected at random had a fair chance of beating the crowd, the idea of the wisdom of crowds would probably never have taken off. Here we address this issue quantitatively by measuring the fraction of individual estimates that are superior to the collective estimate for each forecast experiment. The results are presented in form of histograms and cumulative distributions in figure 5 , where the height of the bars is the proportion of experiments for which that fraction equals $\xi \in[0,1]$. There are a few experiments with $\xi=0$ so that the crowd beats all individuals and one short-range forecast experiment where $85 \%$ of the individuals beat the crowd. The fraction of experiments for which the crowd is more accurate than the majority of the participants is $150 / 205 \approx 0.73$ for the short-range forecasts, $145 / 203 \approx 0.71$ for the medium-range forecasts and $130 / 196 \approx 0.66$ for the long-range forecasts. These figures are obtained by evaluating the cumulative distribution function at $\xi=0.5$. Hence, a randomly chosen individual has probability greater than $1 / 2$ of beating the crowd in about $30 \%$ of the experiments reported here.

We stress that finding that $85 \%$ of the individuals beat the crowd in a particular forecast experiment does not contradict Page's diversity prediction theorem, which asserts that the collective error is always less than the average individual error, i.e., $|\gamma| \leq \epsilon^{1 / 2}$. In fact, that particular experiment involved $N=39$ participants among
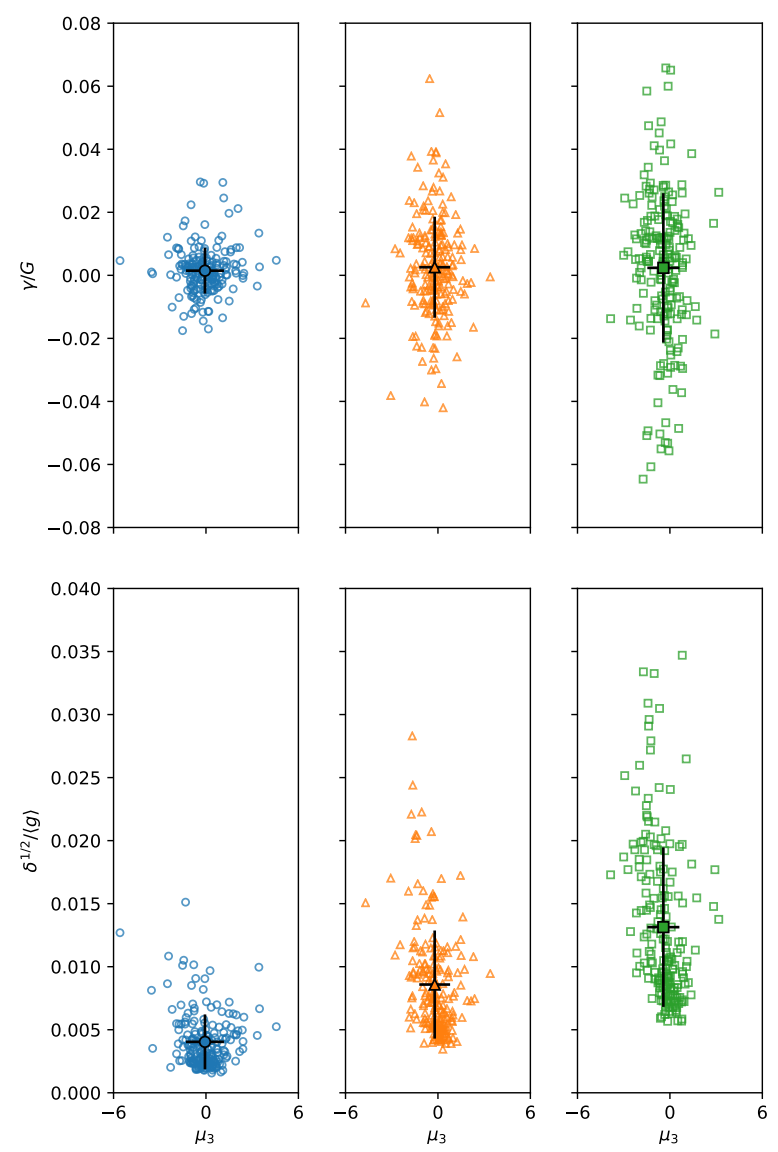

FIG. 4. Scatter plots of the skewness $\mu_{3}$ and the scaled collective error $\gamma / G$ (upper set of panels) and of $\mu_{3}$ and the scaled diversity $\delta^{1 / 2} /\langle g\rangle$ (lower set of panels) for short-range (left panels), medium-range (middle panels) and long-range (right panels) NGDP forecasts. The filled symbols and the horizontal and vertical lines indicate the means and the standard deviations.

which 4 outliers produced completely off the mark estimates resulting in the inflation of the mean individual error. This point betrays the fact that the theorem (5) is largely irrelevant for practical issues concerning the use or not of the crowd as an effective forecaster.

To support these findings, in figure 6 we clump together 8650 experiments of the FRBP forecast database without regard to the economic indicator (there are ten distinct indicators) or to the range of the forecast (there are five distinct ranges). We find that the crowd is superior to any individual in only $1.7 \%$ of the experiments (viz., those for which $\xi=0$ ), whereas it is superior to most individuals in $66.8 \%$ of the experiments (viz., those for which $\xi \leq 1 / 2$ ). Hence the widespread claims about the superiority of the crowd over the individuals 1 is most likely an artifice of selective attention that gives prominence to successful outcomes only. Those claims are legitimate only for unrealistic crowds composed of 

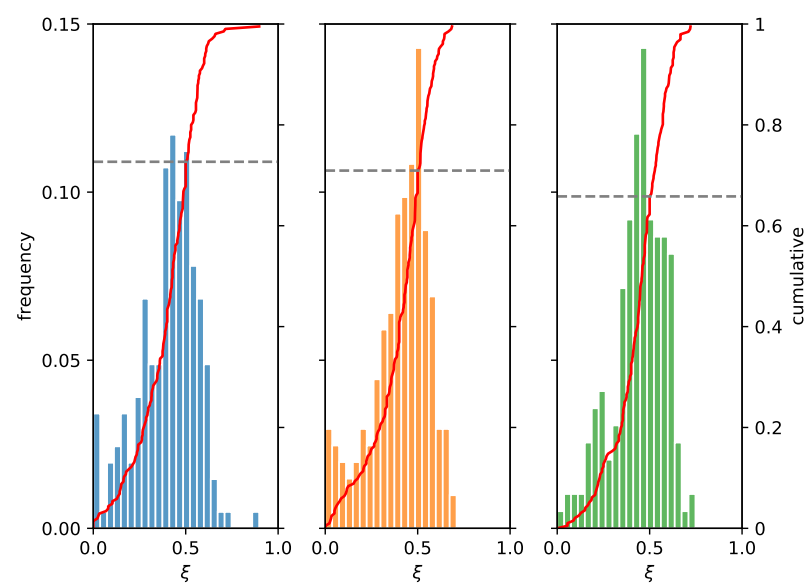

FIG. 5. Histograms of the proportion of experiments for which a fraction $\xi$ of the individual estimates are more accurate than the collective estimate for short-range (left panel), medium-range (middle panel) and long-range (right panel) forecasts. The red curves are the cumulative distributions and the horizontal dashed lines indicate the values of the cumulative distributions at $\xi=0.5$.

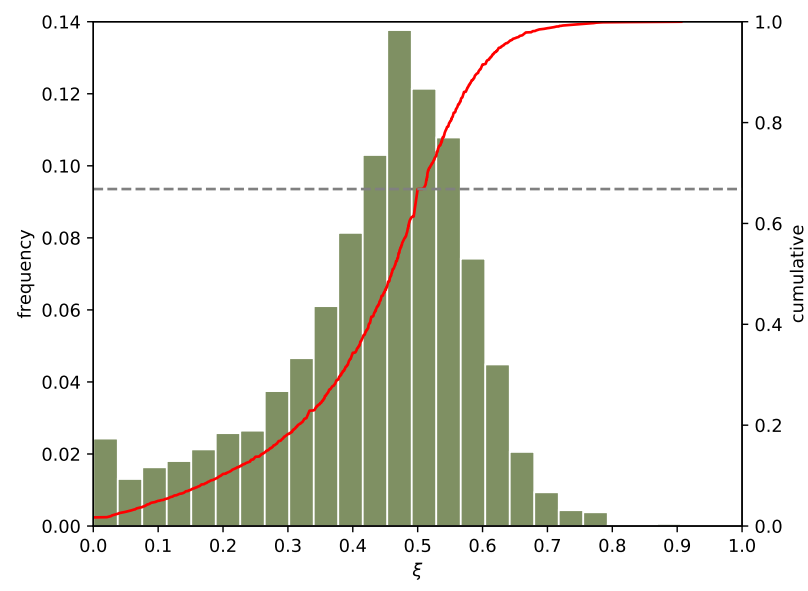

FIG. 6. Histograms of the proportion of experiments for which a fraction $\xi$ of the individual estimates are more accurate than the collective estimate. The data comprises the 8650 experiments of the FRBP forecast database for ten distinct economic indicators and five forecast ranges. The red curve is the cumulative distribution and the horizontal dashed line indicates the value of the cumulative distribution at $\xi=0.5$.

unbiased forecasters, as shown in $\mathrm{V}$

\section{DISCUSSION}

It is almost a cliché to remark that a group of cooperating individuals can solve problems more efficiently than when those individuals work in isolation [18, 19. Cooperation is, in general, a successful problem solving strategy 20, though it is not clear whether it merely speeds up the time to find the solutions, or whether it alters qualitatively the statistical signature of the search for the solutions 21, 22]. Yet, in some cases, cooperation may well lead the group astray resulting in the madness of crowds 23. or, less dramatically, it may simply undermine the benefits of combining independent forecasts 24, 25.

A rather peculiar manner to circumvent the potential negative effects of cooperation while still benefiting from the group intelligence is the so-called wisdom of crowds, i.e., the notion that a collection of independently deciding individuals is likely to predict better than individuals or even experts within the group [1, which ironically seems to have become itself a piece of crowd wisdom [26]. The first report of this phenomenon in the literature was probably Galton's account of the surprisingly accurate estimate of the weight of an ox given by the median of the sample of the individual guesses [2].

Although much of the evidence of the wisdom of crowds is anecdotal (see, e.g., 1, 6]), there are a few efforts aiming at explaining this phenomenon either using a purely statistical rationale [5, 7] or using psychophysical arguments on the nature of the individual estimates 8 . Typically, these approaches aim at inferring the quality of the crowd estimate using information about the distribution of individual estimates (see, e.g., figure 3), such as the variance and the skew of the estimates. Here we address the soundness of those explanations using forecasts of economic indicators from the Federal Reserve Bank of Philadelphia's (FRBP) Survey of Professional Forecasters database [9. The difficulty of the forecasts can be tuned by controlling for the forecast range.

A word is in order about the particularity that the FRBP database comprises the predictions of expert economists, whereas the common view of the wisdom of crowds is that it comprises the predictions of ordinary people. In fact, the wisdom of crowds as a method of information aggregation depends on the presence of an expressive number of experts in the crowd since nothing good can come from the aggregation of random information: if every person in France were asked to specify the gross domestic product of India, the average answer would probably be wildly off $[6$. The widespread interest in the wisdom of crowds stems from the possibility of combining the forecasts of experts in the hope that many expert minds are better than a few. As a matter of fact, just after the publication of Galton's paper on the ox-weighing experiment [2, it was pointed out that Galton had not been exposed to Vox Populi but to Vox Expertorum, as the participants of the contest were butchers and farmers whose livelihood depended on their ability to judge the weight of farm animals before trading 27. Moreover, the 6 pence tickets probably deterred the participation of dilettantes in that celebrated contest.

We stress that the main focus of the research on information aggregation is on the prediction of economic, political and other valuable indicators so that the fore- 
casters are necessarily experts since there is no point in asking ordinary people to predict, say, the NGDP at the end of the current year. Nevertheless, in $\mathrm{V}$ we revisit a few wisdom-of-crowds experiments where the participants are laypeople [14. The experiments are the estimates of the number of candies in a jar, the length of a paper strip, the number of pages of a book and the weight of a bag of beans. These are the kind of factual questions that make sense asking ordinary people. The results generally agree with our findings using the FRBP database. In particular, the probability that a randomly picked participant outperforms the crowd varies from $15 \%$ for the paper-strip experiment to $38 \%$ for the pages-of-a-book experiment, which support our main point that there is a fair chance that random participant will beat the crowd.

Our results suggest that the difficulty of the forecasts is associated with large variances and with long left tails of the distributions of estimates. In addition, we find that a large variance is associated with a poor crowd accuracy, in disagreement with the interpretation of the diversity prediction theorem that the increase of the diversity of the estimates leads to a decrease of the collective error 7]. Moreover, when controlling for the forecast range, we find no evidence of an association between the skew of the estimates and the collective error, in disagreement with the predictions of the augmented quincunx model [8].

We pay special attention to the unbiased estimates assumption that explains the accuracy of the crowd by conjecturing that the errors of the individual estimates spread in equal proportion around the true value of the unknown quantity so that they cancel out when those estimates are combined together [4. In $\mathrm{V}$ we replicate the forecast experiments by replacing the economists by (virtual) unbiased forecasters and show that much of the hailed features of the wisdom of crowds are properties of the combination of unbiased forecasts instead. In that appendix, we also offer a statistical test to check if the economists' forecasts in the FRBP database are in fact biased and find that $85 \%$ of the collective predictions have $p$-value less than 0.05 , which indicates that they are unlikely to be obtained by the aggregation of unbiased forecasts. In addition, in $\mathrm{V}]$ we show that the unbiased estimates assumption cannot be rejected in the case the task is such that the participants are likely to deliver accurate estimates, as in the paper-strip experiment. However, it can safely be rejected in the case that the participants deliver far off estimates as in the candies-in-a-jar experiment. These findings support the view that unbiased forecasters are experts.

The wisdom of real crowds is very different from the apocryphal wisdom of crowds of unbiased forecasters. For instance, the crowd beats all individuals in only around $2 \%$ of the FRBP forecast experiments and it beats most individuals in about $70 \%$ of those experiments, whereas the corresponding figures for the unbiased forecasters are about $16 \%$ and $100 \%$, respectively. Hence, since there is a fair chance that a randomly cho-

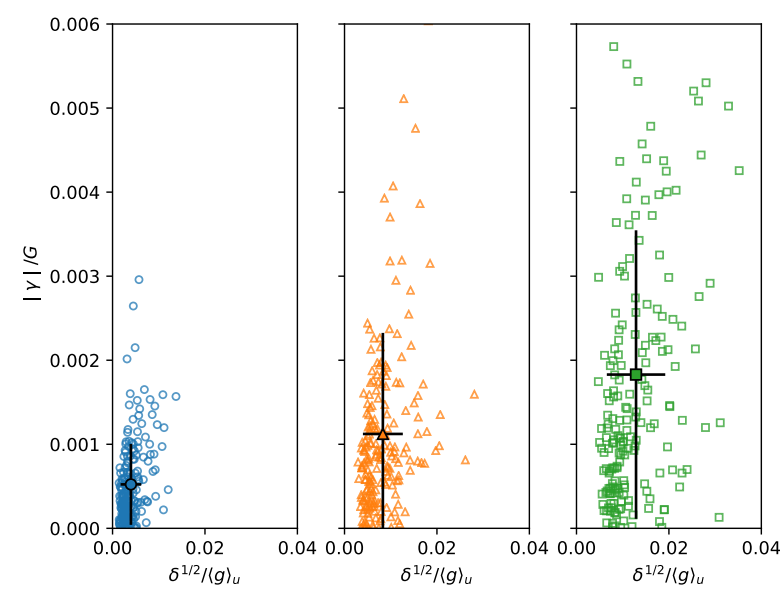

FIG. A1. Scatter plots of the scaled diversity $\delta^{1 / 2} /\langle g\rangle$ and the relative collective error $|\gamma| / G$ for short-range (left panel), medium-range (middle panel) and long-range (right panel) unbiased forecasts. The filled symbols and the horizontal and vertical lines indicate the means and the standard deviations.

sen individual will beat the crowd in real-world forecasts, the only explanation that we can find for the popularity of the wisdom of crowds is selective attention (or cherry picking) that gives prominence to outcomes that tally with Galton's conclusions.

\section{ACKNOWLEDGMENTS}

The research of JFF was supported in part by Grant No. 2020/03041-3, Fundação de Amparo à Pesquisa do Estado de São Paulo (FAPESP) and by Grant No. 305058/2017-7, Conselho Nacional de Desenvolvimento Científico e Tecnológico (CNPq). SMR was supported by the Coordenação de Aperfeiçoamento de Pessoal de Nível Superior - Brasil (CAPES) - Finance Code 001.

\section{APPENDIX A}

In this appendix we examine the predictions of the unbiased estimates assumption for the forecast experiments considered in the main text. In particular, for each experiment with a given number $N$ of participants, we calculate the variance of the estimates $\delta$ and use it to produce $N$ independent unbiased estimates distributed according to a Gaussian of mean $G$ (the true value of the economic indicator) and variance $\delta$, i.e.,

$$
P_{u}\left(g_{i}\right)=\frac{1}{\sqrt{2 \pi \delta}} \exp \left[-\frac{\left(g_{i}-G\right)^{2}}{2 \delta}\right]
$$

for $i=1, \ldots, N$. Hence, by construction, the diversity of the virtual unbiased estimates equals the diversity of 
the economists' forecasts. Although we split the data in three forecast ranges, as done in the main text, we stress that from the perspective of the unbiased forecasters the only difference between those ranges is the variance $\delta$ of the estimates. In addition, we note that for the values of $N$ considered here (see, e.g., figure 1), the arithmetic average $\langle g\rangle_{u}=\sum_{i}^{N} g_{i} / N$ is expected to differ from $G$. In fact, the prediction of the unbiased crowd $\langle g\rangle_{u}$ is a random variable distributed according to the Gaussian

$$
P\left(\langle g\rangle_{u}\right)=\frac{1}{\sqrt{2 \pi \delta / N}} \exp \left[-\frac{\left(\langle g\rangle_{u}-G\right)^{2}}{2 \delta / N}\right]
$$

since the individual estimates $g_{i}$ are statistically independent random variables.

Figure A1 shows the scatter plots of $\delta^{1 / 2} /\langle g\rangle_{u}$ and $|\gamma| / G$ for the unbiased forecasters. As expected, the crowd estimate in this case is about ten times more accurate than in the original forecast experiments. The Spearman correlation coefficient between $\delta^{1 / 2} /\langle g\rangle_{u}$ and $|\gamma| / G$ is $\rho=0.39$ with $p$-value $<10^{-6}$ for the short-range, $\rho=0.37$ with $p$-value $<10^{-6}$ for the medium-range and $\rho=0.44$ with $p$-value $<10^{-6}$ for the long-range forecasts. Hence, the positive and statistically significant correlation between the diversity of the estimates and the crowd accuracy is more pronounced for the unbiased forecasts than for the economists forecasts.

Figure A2 shows the scatter plots of $\mu_{3}$ and $\gamma / G$ as well as of $\mu_{3}$ and $\delta^{1 / 2} /\langle g\rangle_{u}$ for the unbiased forecasters. The noteworthy aspect here is the small range of variation of the skewness values as compared to the results of figure 4. Of course, the nonzero values of $\mu_{3}$ are due to the small number of estimates $N$ in each experiment, since the expected skewness of a Gaussian is zero. The Spearman correlation coefficient between $\mu_{3}$ and $\gamma / G$ is $\rho=-0.09$ with $p$-value $=0.20$ for the short-range, $\rho=0.06$ with $p$-value $=0.35$ for the medium-range and $\rho=0.02$ with $p$-value $=0.73$ for the long-range forecasts. These coefficients are similar to those of the real experiments and their high $p$-values indicate that the skewness of the estimates offers no information on the crowd prediction, regardless of the nature of the forecasters. An unexpected result of our analysis of the economists' forecasts is the negative correlation between the diversity and the skewness of the estimates (see lower set of panels in figure 4), which implies that a large variance is associated with a long left tail of the distribution of estimates. The results of the lower set of panels in figure A2 point to a different conclusion. In fact, the Spearman correlation coefficient between these quantities is $\rho=-0.11$ with $p$-value $=0.10$ for the short-range, $\rho=0.12$ with $p$-value $=0.09$ for the medium-range and $\rho=-0.03$ with $p$-value $=0.64$ for the long-range forecasts. Again, the low values and the inconsistency of the signs of these coefficients as well as their high $p$-values suggest that the skewness plays no role at all on the outcome of unbiased forecasts.

Although the previous scatter plots show only mild quantitative differences between the economists and the
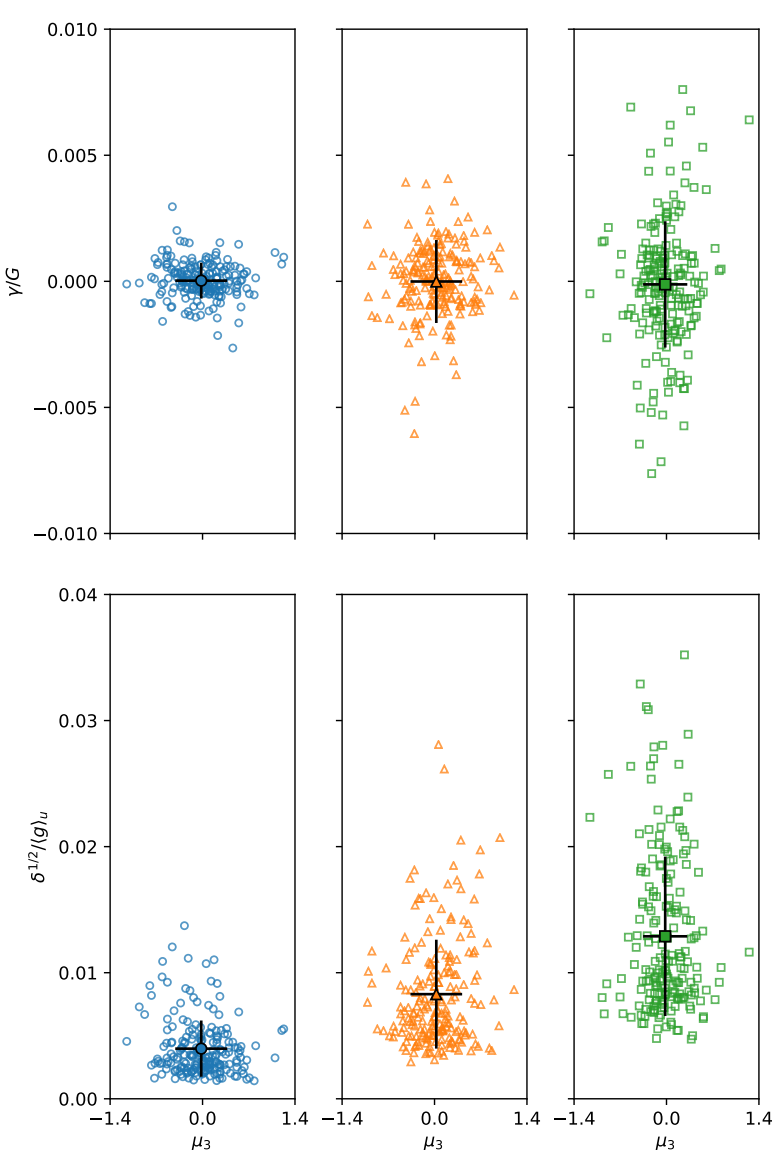

FIG. A2. Scatter plots of the skewness $\mu_{3}$ and the scaled collective error $\gamma / G$ (upper set of panels) and of $\mu_{3}$ and the scaled diversity $\delta^{1 / 2} /\langle g\rangle_{u}$ (lower set of panels) for short-range (left panels), medium-range (middle panels) and long-range (right panels) unbiased forecasts. The filled symbols and the horizontal and vertical lines indicate the means and the standard deviations.

unbiased forecasters, the advantage conferred to the crowd over its members differs starkly between these two types of forecasters. Figure A3, which shows the histograms and the cumulative distributions of the number of experiments for which a fraction $\xi$ of individuals beat the crowd, illustrates this point. In fact, the most probable outcome is that the crowd beats all individuals $(\xi=0)$ in the case of unbiased forecasters, in contrasts to our findings for the human experts (figure 5).

Figure A4 shows the results for the case that all forecasts of the FRBP database are clumped together and replaced by unbiased forecasts. In this case, the crowd is superior to any individual in $16.3 \%$ of the experiments (viz., those for which $\xi=0$ ), whereas it is superior to most individuals in $99.8 \%$ of the experiments (viz., those for which $\xi \leq 1 / 2$ ). Therefore, a crowd of unbiased forecasters exhibits all the exalted attributes of the wisdom of crowds, but a crowd of human experts does not. Hence 

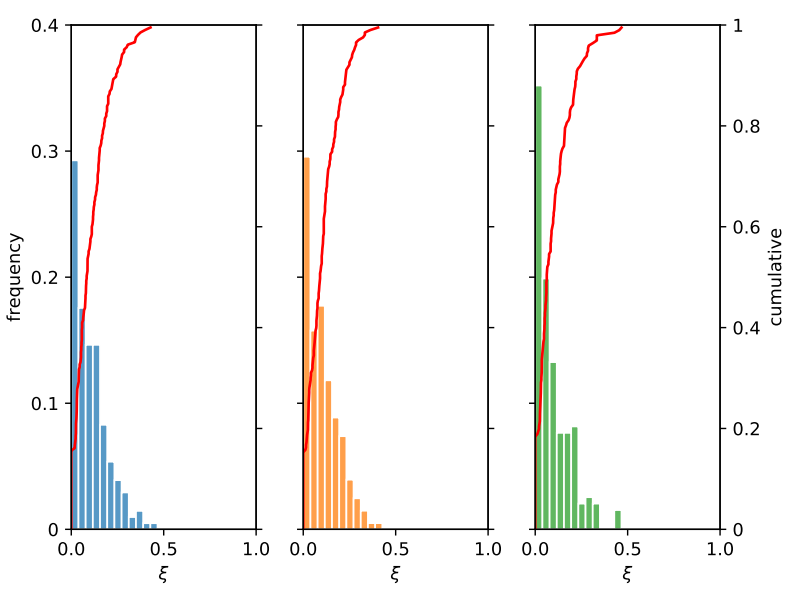

FIG. A3. Histograms of the proportion of experiments for which a fraction $\xi$ of the individual estimates are more accurate than the collective estimate for short-range (left panel), medium-range (middle panel) and long-range (right panel) unbiased forecasts. The red curves are the cumulative distributions.

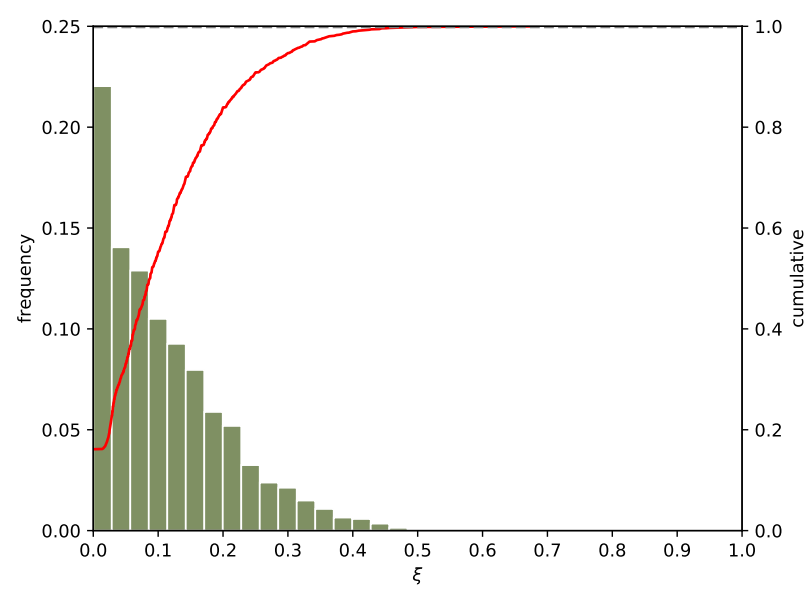

FIG. A4. Histograms of the proportion of experiments for which a fraction $\xi$ of the individual unbiased estimates are more accurate than the collective estimate. The expert forecasts of the 8650 experiments of the FRBP database were replaced by virtual unbiased forecasters. The red curve is the cumulative distribution.

our qualms about the generality and usefulness of that phenomenon.

Although the stark difference between figure 6 and figure A4 offers evidence that the forecasts in the FRBP database are biased, it is worthwhile to seek a more quantitative confirmation that those forecasts are in fact biased. This can be easily achieved if one considers the null hypothesis as the unbiased estimates assumption for which the collective prediction $\langle g\rangle_{u}$ is drawn from the Gaussian distribution A2 . Hence, the probability that the unbiased estimates assumption produces a collective estimate at least as extreme as the collective estimate $\langle g\rangle$

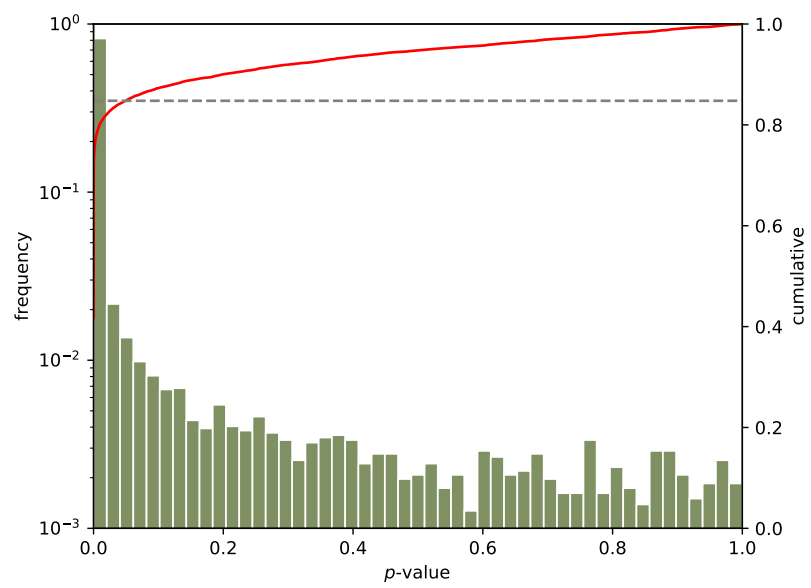

FIG. A5. Histograms of the $p$-values associated with the collective estimates $\langle g\rangle$ for the 8650 experiments of the FRBP database. The null hypothesis is the unbiased estimates assumption, equation A2. The red curve is the cumulative distribution and the horizontal dashed line indicates the value of the cumulative distribution at $p=0.05$.

actually observed in a particular experiment is simply

$$
p=1-\operatorname{erf}\left(\frac{|<g>-G|}{\sqrt{2 \delta / N}}\right),
$$

which is the two-tailed $p$-value. Since there is a $p$-value for each experiment, in figure A5 we present a histogram of the $p$-values associated with the observations $\langle g\rangle$ for the 8650 experiments of the FRBP forecast database. The results show that $85 \%$ of the experiments have $p$ value less than 0.05 , which confers statistical significance to the claim that a significant fraction of the forecasts in the FRBP database are biased.

\section{APPENDIX B}

In this appendix, we analyze briefly four wisdom-ofcrowds experiments that, in contrast to the experiments discussed in the main text, counted with the participation of laypeople only. The experiments are the estimates of the number of candies in a jar, the length of a paper strip, the number of pages of a book and the weight of a bag of beans. The first three experiments are discussed at length in Ref. 14. The data of the four experiments are publicly available in 28. The forecasters are students who have never trained for those kind of guessing tasks and so, in that sense, they can be considered as laypeople. Their skills, however, may vary considerably across the different tasks. Figure B1 shows the histograms of the relative estimates $g_{i} /\langle g\rangle$ with $i=1, \ldots, N$ for those experiments. The number of participants $N$ is different for each experiment, as specified below.

We begin our analysis with the popular wisdom of crowds experiment in which people guess the number of 
candies in a jar. In this particular experiment, $N=105$ students were asked to guess the number of candies in a transparent jar that held $G=636$ candies. The resulting distribution of individual estimates is shown in the upper left panel of figure B1. The collective estimate $\langle g\rangle=531$ is better than $70 \%$ of the individual estimates. The gray region in the histogram indicates the individual estimates that are closer to the true value $G$ than the collective estimate $\langle g\rangle$. The diversity or variance of the estimates is $\delta=48736$. The scaled quantities introduced in the main text are $\delta^{1 / 2} /\langle g\rangle=0.42$ and $\gamma^{1 / 2} / G=0.16$. Using the formulation presented in $\mathrm{V}$, we find that the probability $p$ [see equation (A3)] that a group of $N=105$ unbiased estimators produces a collective estimate at least as extreme as $\langle g\rangle$ is less than $10^{-6}$. Hence, the participants of this experiment are definitely not unbiased estimators.

In the second experiment we discuss here, $N=139$ students were asked to size up a paper strip of length $G=22.4 \mathrm{~cm}$. In contrast to the candies-in-a-jar experiment, we do not expect too far off estimates of the strip length since its true value $G$ is very close to the span unit $(1$ span $=22.86 \mathrm{~cm})$, which is a natural length standard the students were probably aware of. The distribution of individual estimates is shown in the upper right panel of figure B1. The collective estimate is $\langle g\rangle=22.0 \mathrm{~cm}$ and the variance of the estimates is $\delta=12.42 \mathrm{~cm}^{2}$. The scaled diversity is $\delta^{1 / 2} /\langle g\rangle=0.16$ and the scaled collective error is $\gamma^{1 / 2} / G=0.018$. We note that the collective estimate is better than $85 \%$ of the individual estimates and corresponds to a percentage error of only $1.8 \%$. In addition, the probability $p$ that a group of $N=139$ unbiased estimators produces a collective estimate at least as extreme as $\langle g\rangle$ is 0.14 , so we cannot reject the null hypothesis that the forecasts are unbiased. This result supports our point that unbiased forecasters are in fact very skillful estimators.

In the third experiment, $N=97$ students were asked to estimate the weight of a transparent plastic bag containing $G=1.75 \mathrm{~kg}$ of beans. The distribution of individual estimates is shown in the lower left panel of figure B1. The collective estimate is $\langle g\rangle=1.91 \mathrm{~kg}$ and the variance of the estimates is $\delta=0.58 \mathrm{~kg}^{2}$. Hence, the scaled diversity is $\delta^{1 / 2} /\langle g\rangle=0.40$ and the scaled collective error is $\gamma^{1 / 2} / G=0.091$. The collective estimate is better than $84 \%$ of the individual estimates. The probability $p$ that a group of $N=97$ unbiased estimators produces a collective estimate at least as extreme as $\langle g\rangle$ is 0.03 .

Finally, in the fourth experiment, $N=140$ students were asked to estimate the number of pages of a book of $G=784$ pages. The distribution of individual estimates is shown in the lower right panel of figure B1. The collective estimate is $\langle g\rangle=560$ and the variance of the estimates is $\delta=40332$. The scaled diversity is $\delta^{1 / 2} /\langle g\rangle=0.36$ and the scaled collective error is $\gamma^{1 / 2} / G=0.29$, which corresponds to a percentage error of $29 \%$. The collective estimate is better than $62 \%$ of the individual estimates. The probability $p$ that a group of $N=140$ unbiased estimators produces a col-
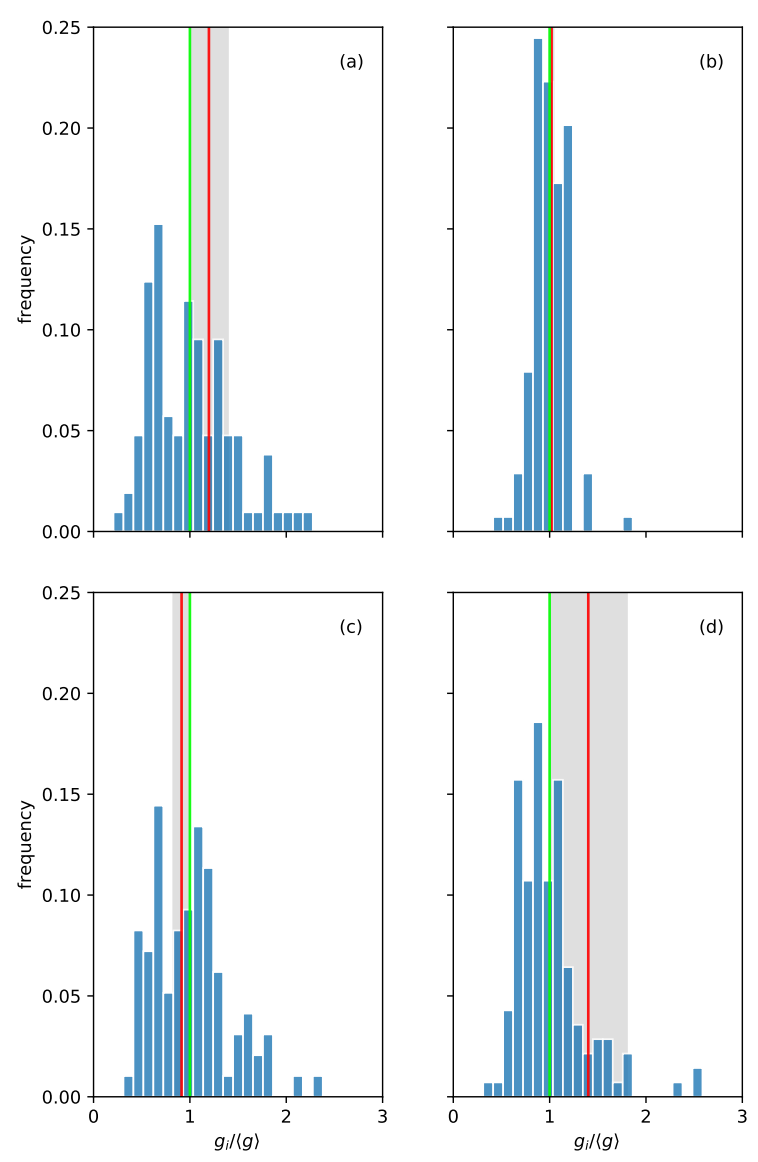

FIG. B1. Histograms of the relative estimates $g_{i} /\langle g\rangle$ for (a) the number of candies in a jar, (b) the length of a paper strip, (c) the weight of a bag of beans and (d) number of pages of a book. The number of participants $N$ is different for each experiment. The vertical red lines indicate the ratio between the true value and the crowd estimate, i.e., $G /\langle g\rangle$, whereas the vertical green lines at $g_{i} /\langle g\rangle=1$ indicate the scaled crowd estimate. The gray regions highlight the individual estimates that are better than the crowd's.

lective estimate at least as extreme as $\langle g\rangle$ is less than $10^{-6}$. Surprisingly, the students turned out to be very bad estimators of the number of pages in a book, perhaps because physical books are no longer part of their lives.

Although in these four experiments the crowd estimate turned out to be better than the estimates of the majority of the participants, in all them there was a fair chance that a randomly chosen participant beat the crowd. For example, in the paper-strip experiment, for which the collective estimate was highly accurate, the crowd was outperformed by $15 \%$ of the individual estimates whereas in the pages-of-a-book experiment it was outperformed by $38 \%$ of the participants. We note that the probability of choosing four experiments at random in the FRBP database such that the crowd beats the majority of the 
forecasters is $(0.7)^{4} \approx 0.24$, so the results of this appendix do not oppose those of the main text.

Picking the candies-in-a-jar and the paper-strip experiments as representative of tasks where, by design, the participants have low and high skills, respectively, we reach the unsurprising conclusion that the less skilled the participants are in a given task, the greater the percentage collective error and the variance of their estimates. This accords with our findings in the main text where the difficulty of the task is associated with the range of the forecasts. In addition, the unbiased estimates assumption (i.e., the null hypothesis) cannot be rejected in the case the task is such that the participants are likely to deliver accurate estimates, as in paper-strip experiment. However, it can safely be rejected in the case that the participants deliver far off estimates as in the candies-ina-jar experiment. Again, these findings agree with the view of unbiased forecasters as ultimate experts.
[1] Surowiecki J 2004 The Wisdom of Crowds: Why the Many Are Smarter than the Few and How Collective Wisdom Shapes Business, Economies, Societies, and Nations (New York: Doubleday and Company)

[2] Galton F 1907 Vox Populi Nature 75450

[3] Wallis K F 2014 Revisiting Francis Galton's Forecasting Competition Stat. Sci. 29420

[4] Bates J M and Granger C W J 1969 The combination of forecasts Oper. Res. Q. 20451

[5] Armstrong J S 2001 Combining Forecasts in Principles of forecasting: a handbook for researchers and practitioners, edited by Armstrong J S (Dordrecht: Kluwer Academic Publishing) pp. 417-439

[6] Sunstein C 2006 Infotopia: How Many Minds Produce Knowledge (New York: Oxford University Press)

[7] Page S E 2007 The Difference: How the Power of Diversity Creates Better Groups, Firms, Schools, and Societies (Princeton, NJ: Princeton University Press)

[8] Nash U W 2014 The Curious Anomaly of Skewed Judgment Distributions and Systematic Error in the Wisdom of Crowds PLoS ONE 9 e112386

[9] https://www.philadelphiafed.org/research-anddata/real-time-center/survey-of-professional-forecasters

[10] Fontanari J F 2016 When more of the same is better EPL 11328009

[11] Tan P-N, Steinbach M and Kumar V 2014 Introduction to Data Mining (Essex: Pearson Education Limited)

[12] Price G R 1970 Selection and Covariance Nature 227520

[13] Frank S A 2012 Natural selection. IV. The Price equation J. Evol. Biol. 251002

[14] Nobre D A and Fontanari J F 2020 Prediction diversity and selective attention in the wisdom of crowds arXiv:2001.10039v2
[15] Stigler S M 1989 Francis Galton's account of the invention of correlation Stat. Sci. 473

[16] Vul E and Pashler H 2008 Measuring the crowd within: Probabilistic representations within individuals Psychol. Sci. 19645

[17] Mauboussin M J 2012 The Success Equation: Untangling Skill and Luck in Business, Sports, and Investing (Cambridge, MA: Harvard Business Review Press)

[18] Huberman B A 1990 The performance of cooperative processes Physica D 4238

[19] Clearwater S H, Huberman B A and Hogg T 1991 Cooperative Solution of Constraint Satisfaction Problems Science $\mathbf{2 5 4} 181$

[20] Bloom H 2001 Global Brain: The Evolution of Mass Mind from the Big Bang to the 21st Century (New York, Wiley)

[21] Reia S M, Amado A C and Fontanari J F 2019 Agentbased models of collective intelligence Phys. Life Rev. $\mathbf{3 1}$ 320

[22] Reia S M, Aquino L F and Fontanari J F 2020 The surprising little effectiveness of cooperative algorithms in parallel problem solving Eur. Phys. J. B 93140

[23] MacKay C 1841 Extraordinary Delusions and the Madness of Crowds ( London: Richard Bentley)

[24] King A J, Cheng L, Starke S D and Myatt J P 2011 Is the true 'wisdom of the crowd' to copy successful individuals? Biol. Lett. 8197

[25] Lorenz J, Rauhut H, Schweitzer F and Helbing D 2011 How social influence can undermine the wisdom of crowd effect Proc. Natl Acad. Sci. USA 1089020

[26] Prelec D, Seung H S and McCoy J 2017 A solution to the single-question crowd wisdom problem Nature $\mathbf{5 4 1} 532$

[27] Perry-Coste FH 1907 The ballot-box Nature 75509

[28] https://github.com/JoseFontanari/Wisdom_of_Crowds 